\title{
Wie kommt das Haben zum Soll?
}

\section{Werner Bauer}

Dr. med., Präsident des Schweizerischen Instituts für ärztliche Weiter- und Fortbildung SIWF

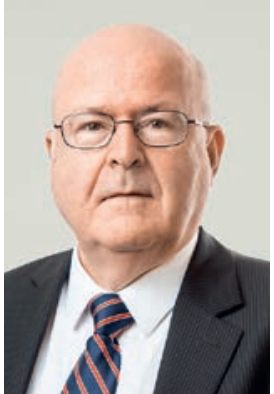

"Jedes Mal, wenn wir daran waren, Gemeinschaften zu bilden, wurden wir umorganisiert. Später im Leben habe ich gelernt, dass wir dazu neigen, neuen Situationen mit Reorganisation zu begegnen: Und dies kann eine glänzende Methode sein, die Illusion von Fortschritt zu schaffen, während Verwirrung, Wirkungslosigkeit und Demoralisierung produziert werden.»

Titus Petronius Arbiter (ca. 14 bis 66 n. Chr.)

"Take me to the magic of the moment on a glory night, where the children of tomorrow share their dreams with you and me. Take me to the magic of the moment on a glory night, where the children of tomorrow dream away in the wind of change.»

Scorpions, Wind of Change (1990)

Weiterfahren wie bisher oder Veränderung suchen, wenn sich bedeutsame Entwicklungen abzeichnen? Als Individuen und in Institutionen haben wir die Tendenz, vorerst zu beobachten und abzuwarten. Oft aber lösen Konflikte und drängende Fragen auch den Aktivismus aus, den Petronius Arbiter schon vor 2000 Jahren beklagte. Umstrukturierungen und Reorganisationen mögen je nach Fall zwingend oder sinnvoll sein, erfolgreich sind sie nur, wenn sie am Kern der Problematik ansetzen und ihn nicht nur übertünchen.

Was haben nun Soll, Haben, Weiterfahren oder Verändern mit dem Artikel über die Verteilung der Anzahl Ärztinnen und Ärzte in Weiterbildung in dieser Ausgabe der Schweizerischen Ärztezeitung zu tun? Die Ergebnisse der Umfrage zu heute besonders aktuellen "Evergreens», welche die ETH 2017 im Auftrag des SIWF bei allen Assistenzärztinnen und -ärzten und bei den Weiterbildungsstättenleitern durchgeführt hat, liefern Stoff zum Nachdenken, ob Handlungsbedarf besteht. Die Antworten sind subjektiv und basieren auf individueller Erfahrung, aber in ihrer Gesamtheit sind sie doch eine ernst zu nehmende Meinungsäusserung aus den schweizerischen Weiterbildungsstätten. Sie vermitteln auch ein Echo auf Gedankenspiele, die in letzter Zeit vor allem in der öffentlichen Verwaltung erstaunlich beliebt geworden sind. Es geht um die aktive Steuerung und Verkürzung der Facharztweiterbildung und um die Forderung, der ärztliche Nachwuchs müsse sich früher als heute definitiv für eine Fachrichtung entscheiden.
Die Beantwortung der Frage nach der Wünschbarkeit einer gezielten Steuerung der Facharztweiterbildung zeigt, dass die Frage, wie viele Ärzte für die Deckung des zukünftigen Bedarfs an ärztlichen Leistungen in den verschiedenen Fachbereichen benötigt werden, als berechtigt wahrgenommen wird. Trotzdem findet ein Vorschlag, der in Richtung konkreter Stellenplanung geht, keine Mehrheit. Dabei zeigen sich Unterschiede zwischen Landesteilen und Fächern, indem die französischsprachige Schweiz und die Orthopädische Chirurgie je "steuerungsfreudiger» sind als die anderen Regionen und Disziplinen. Beim Zurückkommen auf die Titelfrage «Soll und Haben» muss festgestellt werden, dass wir nur dann vom Haben zu einem sinnvollen Soll kommen können, wenn wir dieses in Form des zu erwartenden Bedarfs genügend genau bestimmen könnten. Dies ist nicht einfach, wäre aber die Voraussetzung für eine Veränderung, die wirklich eine Verbesserung bringt.

Bei der Frage nach dem zumutbaren Zeitpunkt des Entscheides für einen Facharzttitel spricht sich die Mehrheit für eine liberale Haltung aus, auch wenn durch eine frühere Festlegung die Weiterbildungszeit etwas verkürzt werden könnte. Ein Zwang zum Entscheid schon im Studium findet kaum Unterstützung, ein erstes und auch noch ein zweites "Lehr- und Wanderjahr» wird als sinnvoll beurteilt, auch wenn dieser Ausdruck neulich in Verwaltungskreisen negativ besetzt verwendet wurde.

Die bejahenden Antworten auf die Frage nach der Anstellung von ärztlichen Mitarbeitenden, die für die Betriebsführung benötigt werden, ohne dass effektiv genügende Ressourcen für deren Weiterbildung zur Verfügung stehen, stimmen nachdenklich und rufen nach dem "wind of change». Sie dokumentieren eine gewisse Schwäche des jetzigen liberalen Systems, die dazu führen kann, dass sich in bestimmten Fachgebieten die Stellenzahl primär am betrieblichen Bedarf und weniger am Bedarf des Gesundheitswesens orientiert. Diese Tatsache muss ernst genommen werden und ruft sicher nach Massnahmen zur Sicherstellung der finanziellen Ressourcen und der zeitlichen Freiräume sowie zum Abbau administrativer Belastungen an den Weiterbildungsstätten. Weitergehende Lenkungsmassnahmen wären nur dann sinnvoll und akzeptabel, wenn davon ausgegangen werden könnte, dass sie wirklich vom «Soll» zum «Haben» führen. 